\title{
The HopX (AvrPphE) Family of Pseudomonas syringae Type III Effectors Require a Catalytic Triad and a Novel N-Terminal Domain for Function
}

\author{
Zachary L. Nimchuk, ${ }^{1}$ Emily J. Fisher, ${ }^{1}$ Darrell Desveaux, ${ }^{1}$ Jeffery H. Chang, ${ }^{1}$ and Jeffery L. Dangl,2,3,4 \\ ${ }^{1}$ Department of Biology, ${ }^{2}$ Curriculum in Genetics, ${ }^{3}$ Department of Microbiology and Immunology, ${ }^{4}$ Carolina Center \\ for Genome Sciences, University of North Carolina, Chapel Hill, NC 27599, U.S.A.
}

Submitted 11 September 2006. Accepted 27 October 2006.

\begin{abstract}
Many gram-negative plant pathogenic bacteria employ type III secretion systems to deliver effector proteins directly into the host cell during infection. On susceptible hosts, type III effectors aid pathogen growth by manipulating host defense pathways. On resistant hosts, some effectors can activate specific host disease resistance $(R)$ genes, leading to generation of rapid and effective immune responses. The biochemical basis of these processes is poorly understood. The HopX (AvrPphE) family is a widespread type III effector among phytopathogenic bacteria. We determined that HopX family members are modular proteins composed of a conserved putative cysteine-based catalytic triad and a conserved potential target/cofactor interaction domain. HopX is soluble in host cells. Putative catalytic triad residues are required for avirulence activity on resistant bean hosts and for the generation of a cell-death response in specific Arabidopsis genotypes. The putative target/cofactor interaction domain is also required for these activities. Our data suggest that specific interaction with and modification of a cytosolic host target drives HopX recognition in resistant hosts and may contribute to virulence in susceptible hosts. Surprisingly, the Legionella pneumophila genome was found to contain a protein with similarity to HopX in sequence and domain arrangement, suggesting that these proteins might also contribute to animal pathogenesis and could be delivered to plant and animal hosts by diverse secretion systems.
\end{abstract}

Additional keyword: bacterial virulence.

The majority of plant-microbe interactions do not result in disease. This is due to a sophisticated immune system that allows plants to recognize microbial elicitors and activate a complex array of defense responses. Yet pathogens by definition can avoid or dampen this basal defense system to colonize host

Corresponding author: J. L. Dangl; E-mail: dangl@email.unc.edu; Fax: +1.919 .962 .5624 .

Current address of Z. Nimchuk: California Institute of Technology, Division of Biology, 156-29, Pasadena, CA 91125 USA.

Current address of D. Dexveaux: Department of Cell and Systems Biology, University of Toronto, 25 Willcocks Street M5S 3B2, Toronto, ON, Canada.

Current address of J. H. Chang; Department of Botany \& Plant Pathology, Oregon State University, Corvallis, OR 97331, U.S.A. plants effectively. Phytopathogenic bacteria accomplish this by delivering a suite of 15 to 30 protein effectors into the host cell via a type III secretion system (T3SS) (Grant et al. 2006; Mudgett 2005). Collectively, these T3SS effectors contribute to pathogen virulence. Conversely, single T3SS effectors are sufficient, in specific host genotypes, to trigger the action of plant disease resistance $(R)$ genes (Nimchuk et al. 2003). In these instances, the particular T3SS effector in question renders the bacterial strain avirulent; these were originally termed avirulence (Avr) proteins.

The majority of $\mathrm{R}$ proteins belong to the NB-LRR superfamily and contain a central nucleotide binding site (NB), Cterminal leucine rich repeats (LRR) of variable length, and either an N-terminal domain that encodes either a coiled-coil domain similarity to the intracellular signaling domain of toll interleukin 1 receptor (TIR). NB-LRR protein activation triggers a complex suite of defense pathway activation and is frequently accompanied by a form of programmed cell death termed the hypersensitive response (HR). There has been speculation that NB-LRR proteins act as direct receptors for Avr proteins, with the LRR domains governing recognition specificity. While some NB-LRR proteins appear to function in this manner (Dodds et al. 2006), clear evidence suggests that other NB-LRR proteins detect Avr proteins indirectly. In this model, termed the "guard hypothesis," the NB-LRR protein is associated with a host protein that is a target for a given T3SS effector virulence factor (Chisholm et al. 2006; Dangl and Jones 2001). In these examples, the interaction of a T3SS effector and its host cellular target activates the corresponding NB-LRR proteins in a manner not yet well understood. Thus, at least some NB-LRR proteins appear to guard specific targets of type III effectors and to respond subsequently to specific activation signals (Belkhadir et al. 2004b). The guard hypothesis also implies that, in the absence of the particular NB-LRR protein, interaction with a target protein provides some benefit to the pathogen. Available evidence suggests that most effectors have multiple targets inside plant host cells (Belkhadir et al. 2004a; Chisholm et al. 2006; Dangl and Jones 2001).

Suppression of host defenses is a common function for T3SS effectors in susceptible ( $r$ ) plants (Nomura et al. 2005). This effect is often seen following expression of a given effector as a transgene within susceptible host plants (He et al. 2006). In contrast, mutation of a single T3SS effector in a particular bacterial strain often yields no loss of pathogen virulence, presumably due to effector redundancy. This confounds assignment of virulence function to T3SS effectors, as does the lack 
of obvious homology of most T3SS effectors to known proteins. In light of these problems, the use of comparative genomics, crystallography, and molecular modeling has allowed assignment of biochemical functions to several T3SS effectors. For example, several families of effector proteins encode cysteine proteases of different specificities, one family encodes a tyrosine phosphatase and another, a novel E3 ligase (Desveaux et al. 2006; Grant et al. 2006; Mudgett 2005). The respective enzymatic activities of these T3SS effectors are correlated with their ability either to trigger specific NB-LRR protein function on resistant hosts, to contribute to virulence in susceptible plants, or both.

AvrPphE (recently renamed HopX) (Lindeberg et al. 2005) is a T3SS effector protein originally identified in Pseudomonas syringae pv. phaseolicola strains by its ability to trigger $R 2$ disease-resistance function in some bean cultivars (Mansfield et al. 1994). HopX alleles are found in diverse $P$. syringae pv. phaseolicola strains, and some are variant alleles that do not trigger R2 (Stevens et al. 1998). Subsequent genomics studies have uncovered hopX family members in diverse $P$. syringae strains as well as in unrelated T3SS-harboring phytopathogenic bacteria such as Ralstonia solanacerum and Xanthomonas campestris pathovars (Buell et al. 2003; Charity et al. 2003; da Silva et al. 2002; Deng et al. 2003; Rohmer et al. 2003; Salanoubat et al. 2002). The widespread distribution of hopX family members in diverse pathogenic bacteria suggests that it plays a conserved role in virulence on a wide range of plant hosts.

Here, we demonstrate that HopX family members are modular proteins that contain a putative cysteine-based catalytic triad and a potential protein-cofactor interaction platform. The putative catalytic triad consists of cysteine, histidine, and aspartic acid and is similar to that utilized by diverse enzyme families including cysteine proteases, peptide $\mathrm{N}$-glycanases (PNGase), and phytochelatin synthase (Makarova et al. 1999), which are all members of the transglutaminase (TGase) protein superfamily. Cysteine proteases, as noted above, mediate proteolytic cleavage of target plant proteins. PNGase interacts with the proteosome via a PUB (peptide: $N$-glycanase/UBA or UBX-containing proteins) domain and catalyzes the deglycosylation of misfolded glycoproteins intended for degradation by the proteosome (Hirsch et al. 2003; Li et al. 2005; Suzuki et al. 2002). Phytochelatin synthase catalyzes the generation of phytochelatin, a polymer of heavy metal-binding thiol peptides, from glutathione and is involved in detoxification of heavy metals in diverse species (Rea 2006; Romanyuk et al. 2006). TGases are enzymes that can either cross-link proteins or amidate specific residues on target proteins (Lorand and Graham 2003). While these enzyme classes seem disparate, they utilize a similar catalytic mechanism.

We demonstrate that both the putative catalytic triad and a novel conserved $\mathrm{N}$-terminal domain are required for HopX to trigger R2-dependent disease resistance on bean and also for the ability of a HopX allele from $P$. syringae pv. tomato DC3000 to trigger a cell-death response in Arabidopsis. This cell-death response is polymorphic among Arabidopsis accessions, suggesting polymorphism in a host target. A requirement for the catalytic triad was demonstrated for several HopX family members using assays on both bean and Arabidopsis. HopX $_{\text {Pto DC3000 }}$ is localized in the cytoplasm of host cells, suggesting that the HopX family modifies a cytoplasmic host target. Lastly, using sequence identity and domain arrangement, we found that the animal pathogen Legionella pneumophila contains a HopX-like protein that has the potential to be a virulence effector. Our findings open the opportunity to identify targets for HopX using the Arabidopsis genetic model system and add to the observation that animal and plant bacterial pathogens may utilize similar virulence mechanisms delivered by disparate secretion systems.

\section{RESULTS}

\section{HopX family members and a Legionella protein encode modular proteins with a putative catalytic triad domain and a potential protein/cofactor interaction platform.}

The HopX $\mathrm{X}_{\text {Pto DC3000 }}$ sequence was used to search the NCBIdatabase using the PSI-BLAST program (Altschul et al. 1997) and to collect homologs from other phytopathogenic bacteria. Several HopX family members were identified in our searches.

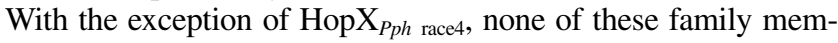
bers are known to trigger any $R$ response when delivered from their parent strain to its respective host plant. During these searches, we noted that the central region of HopX $\mathrm{X}_{\text {Pto DC300o }}$ was strikingly homologous to the conserved catalytic triad domain of eukaryotic PNGase (Fig. 1A). PNGases are members of the TGases superfamily that all utilize a cysteine-histidine-aspartic acid-based catalytic triad for enzymatic activity. Alignment of several $P$. syringae HopX family members with the catalytic triad of diverse eukaryotic PNGases demonstrated that this conservation was not limited to the HopX $\mathrm{X}_{\text {Pto DC3000 }}$ allele (Fig. 1A). There was absolute conservation of the catalytic triad residues between HopX family members and PNGases (Fig. 1A, red dots) as well as conservation of amino acids adjacent to the catalytic triad cysteine and histidine. Secondary structure prediction of the putative catalytic triad of the HopX family was strikingly similar to the known structure of the PNGase catalytic triad in which the active site cysteine is located at the beginning of an alpha helix, and the histidine and aspartic acid residues are located following beta sheets (Fig. 1B). We did not detect homology between HopX and PNGase or any of the other TGase superfamily enzyme classes outside of the catalytic core.

HopX homologs are found in diverse phytopathogenic bacteria. We hypothesized that these family members were active as virulence factors and that amino-acid residues critical for virulence, including the catalytic triad residues, would be maintained across the family. Multiple alignment revealed broad divergence across the HopX family but also revealed the maintenance of the putative catalytic triad residues (Fig. 1C, red dots) and a novel conserved domain $\mathrm{N}$-terminal to the putative triad (Fig. 1C, A domain, red line). The first 30 amino acids of T3SS effectors are sufficient to direct export of reporter proteins in many pathogen bacteria (Sorg et al. 2005). Importantly, neither of these domains overlapped with the T3SS export sequence at the N-terminus of HopX family members.

Using iterative BLAST searches, we identified Lpg2408 from Legionella pneumophila, the causal agent of Legionnaires' disease (Cianciotto 2001), which shared both sequence homology and conserved domain arrangements with HopX (Fig. 1C). $L$. pneumophila lacks a T3SS, suggesting that these domains were important for functions unrelated to T3SS export. Lpg2408 is nearly identical in each of three sequenced L. pneumophila strains, Lens (Lpl2331), Philadelphia 1 (Lpg2408), and Paris (Lpp2475) (data not shown). Residues within the A domain that are conserved among all HopX alleles are also invariant in Lpg2408, including Asn124 and Asp126 from HopX $X_{\text {Pto DC3000 }}$ (Fig. 1C). Residues conserved in the putative catalytic domain included the catalytic triad as well as residues adjacent to the putative catalytic cysteine and aspartic acid (Fig. 1C). Residues adjacent to the putative active site histidine were less conserved (Fig. 1C). Enhanced variability around the active site histidine relative to the other catalytic residues is a common feature among HopX alleles (Fig. 1C) and could reflect a role for this amino acid in active site stabilization rather than substrate interaction (Makarova et al. 1999). It is, hence, likely that His 112 of 
Lpg2408 is a putative catalytic residue in this protein. Secondary structure prediction on Lpg2408 suggested a similar helix-sheetsheet-sheet arrangement within the proposed catalytic core, as seen in HopX and PNGase (data not shown). In this case, H112 of $\operatorname{Lpg} 2408$ was positioned at the beginning of the second $\beta$ sheet, consistent with it being a catalytic residue (data not shown). Additionally, a putative Ralstonia solanacerum effector (NP_523141) was identified in iterative PSI-Blast searches with HopX that also possessed an Lpg2408-like His catalytic residue (data not shown). The physical arrangement of the catalytic triad residues are conserved within the TGase superfamily, however the spatial distances between them are variable. This is apparently true of the HopX alleles as well (Fig. 1C) (Makarova 1999). The Lpg2408 C-terminus did not align with the region of HopX C-terminal to the catalytic domain (data not shown). Overall comparison of the sequence relatedness between Lpg2408 and the HopX revealed that Lpg2408 is most closely related to the Xanthomonas and Ralstonia HopX homologs (data not shown). The $P$. syringae HopX allele most closely related to Lpg2408 over their respective full-length alignments was HopX $_{P_{s m} 4326}$ (Fig. 1C), while HopX ${ }_{P S d}$ PDDCC526 was the most closely related $P$. syringae sequence (data not shown) when just the A domain and catalytic triad were considered.

Despite the strong homology and conservation of PNGaselike catalytic triad residues in HopX family members, we were unable to detect any in vitro enzyme activity on commonly tested substrates for any of the TGase superfamily enzymes classes using various HopX members. The lack of detectable in vitro activity using general substrates is not surprising. Most T3SS effectors from pathogenic bacteria encoding cysteine proteases are active in vitro only when an in vivo host target is used as a substrate or following processing of a proenzyme or both (Axtell et al. 2003; Coaker et al. 2005; Shao et al. 2003). The cysteine protease activity associated with the $P$. syringae T3SS effector HopPtoN on general substrates in vitro is orders of magnitude weaker than positive control standards, indicating that even this effector operates suboptimally in vitro (LopezSolanilla et al. 2004). Thus, the HopX family may have evolved highly specific substrate selectivity as a common feature among them. Similar domain searches with the N-terminal "domain A" (Fig. 1C) did not reveal obvious homology to known proteins (discussed below). Specific deletion of domain A did not result in a gain of in vitro enzyme activity, indicating that this domain does not negatively regulate the catalytic domain, at least on common TGase and cysteine protease substrates (data not shown).

\section{The putative HopX catalytic triad is required for $R 2$ function on bean.}

In the absence of in vitro enzyme activity, we sought genetic evidence for the functional relevance of the conserved catalytic triad of HopX family members. We substituted alanine resi-

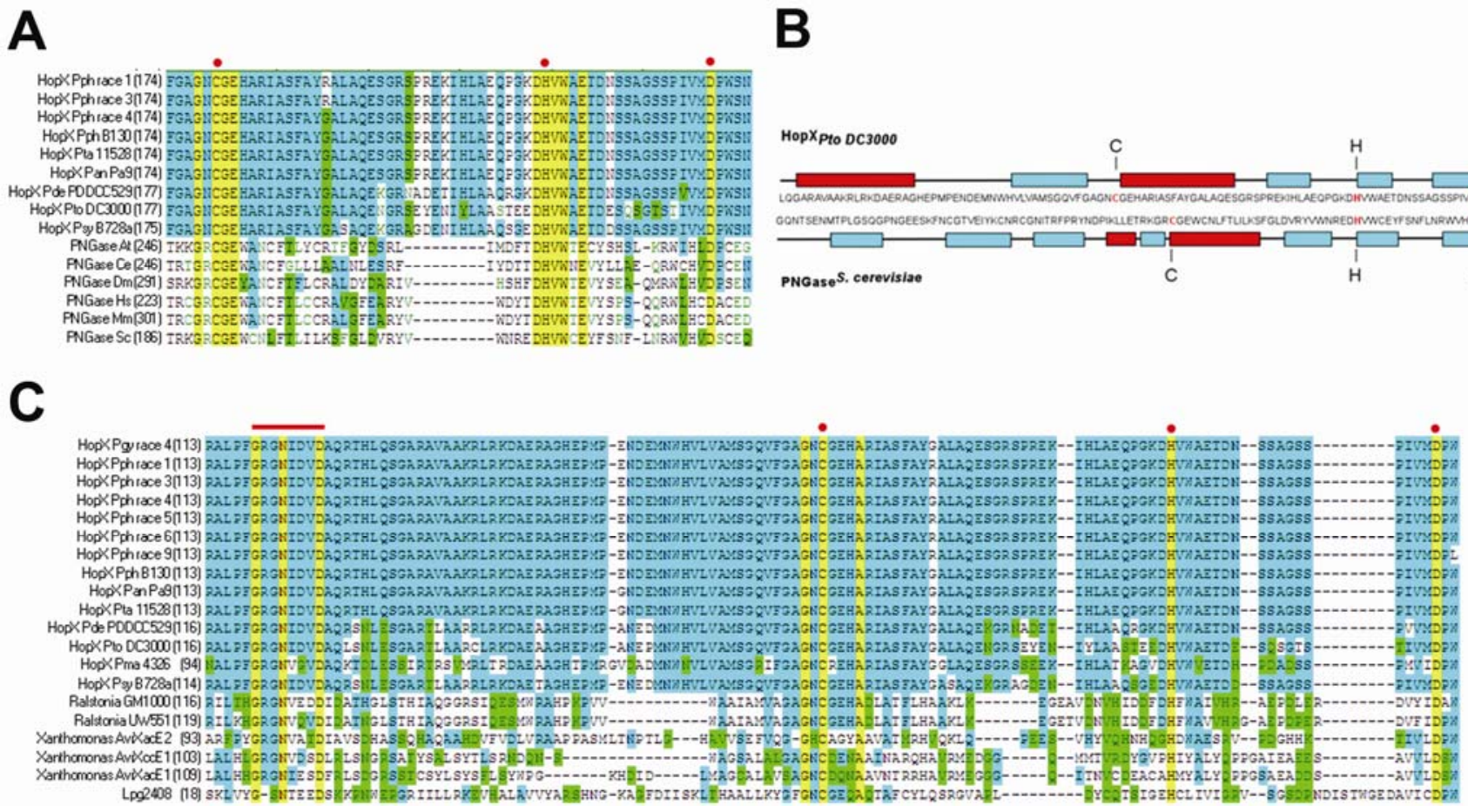

Fig.1. HopX family members contain a consensus catalytic triad similar to that found in the transglutaminase superfamily and a conserved N-terminal domain. A, Homology between the putative catalytic triad of Pseudomonas syringae HopX alleles and the known catalytic triad of eukaryotic PNGases (At $=$ Arabidopsis thaliana $; \mathrm{Ce}=$ Caenorhabditis elegans; $\mathrm{Dm}=$ Drosophila melanogaster $; \mathrm{Mm}=$ Mus musculus $; \mathrm{Sc}=$ Saccharomyces cerevisiae $; \mathrm{Hs}=$ Homo sapiens. $\mathrm{Red}$ dots indicate the catalytic triad of PNGase. B, Secondary structure prediction of the putative catalytic triad of the HopX family compared with the known secondary structure of S. cerevisiae PNGase. Putative catalytic residues (red in the sequences; bold black above and below domains) are identified in relation to $\alpha$-helical (red) or $\beta$-sheet (blue) elements. Note the lack of secondary structure conservation upstream of the catalytic triad. C, Conservation of the putative catalytic residues (red dots) and the A domain (red line) between divergent HopX homologs and Lpg2408 from the strain Legionella pneumophila Philadelphia 1 (bottom). Sequences were downloaded from the National Center for Biotechnology Information and were aligned using the Align-X function of Vector NTI. Abbreviations refer to the pathogen species, the Pseudomonas syringae pathovar, or both. GenBank numbers for bacterial species and pathovars: $P$. syringae pv. tabaci (Pta) 11528, AAP23130; P. syringae pv. phaseolicola (Pph) race 4, AAA67930; Pph B130, AAP23127; P. syringae pv. angulata (Pan) Pa9, AAP23110; P. syringae pv. syringae (Psy) B728a, AAF71495; P. syringae pv. delphinii (Pde) PDDCC529, AAP23116; P. syringae pv. tomato (Pto) DC3000, AAO59038; P. syringae pv. maculicola (Psm) 4326, ABA41434; P. syringae pv. glycinea (Pgy), AAP23121; Ralstonia solanacearum GM1000, NP_521488; R. solanacearum UW551, ZP_0094658.1; Xanthomonas axonopodis pv. citri 306, AvrXacE2, NP_643532; X. axonopodis pv. citri 306, AvrXacE1, NP_640642; X. axonopodis pv. citri 306, AvrXccE1, NP_644739. Note that other alleles from various $P$. syringae pv. phaseolicola races are not in the database but were reconstructed from Stevens and associates (1998). Color code: white $=$ nonconserved residue, yellow $=$ identical, blue $=$ highly conserved, and green $=$ weakly conserved. A and C, Numbers in parentheses refer to the position of the first residue displayed for each HopX allele. 
dues for the putative conserved catalytic amino acids of HopX, C179A, H215A, and D233A. We tested the ability of $\mathrm{HopX}_{P p h \text { race4 }}$ and these mutant derivatives to trigger $R 2$-mediated resistance in bean. Wild-type and mutant hop $X_{P p h \text { race } 4}$ constructs were expressed behind a dual lac-nptII promoter on a broad-host range plasmid (Kovach et al. 1995) and were mobilized into $P$. syringae pv. phaseolicola race 6 , which by itself does not trigger any known $R$ function on bean (Stevens et al. 1998). Bacterial strains expressing the mutant and wild-type genes were inoculated into pods of bean cultivar A43 (R2) to assess their ability to elicit the HR, a hallmark of a diseaseresistance response. $P$. syringae pv. phaseolicola race 6 carrying an empty vector caused water soaking lesions 3 days postinoculation (dpi); an expected disease-susceptible response (Stevens et al. 1998). In contrast, bacteria expressing the wildtype $h o p X_{P p h \text { race4 }}$ elicited a robust HR within $24 \mathrm{~h}$ on A43. Strains with mutations in the proposed catalytic residues of
HopX failed to elicit the R2-mediated HR (Fig. 2A). Interestingly, the D233A mutant did not completely abolish the HR. Significant "browning" indicative of the HR was observed 3 dpi (not shown). An HA-epitope tag was incorporated at the Cterminus of the wild-type and catalytic triad hop $X_{P p h \text { race4 }} \mathrm{mu}$ tant alleles in order to monitor protein levels. Unlike C179A and D233A, the H215A mutant of HopX accumulated to significantly lower levels in cells of $P$. syringae pv. phaseolicola race 6 (Fig. 2B).

We next measured the growth of $P$. syringae pv. phaseolicola race 6 strains carrying each hopX variant in bean leaves (Fig. 2C). The $P$. syringae pv. phaseolicola race 6 strain carrying an empty vector grew robustly over 3 days on A43. Bacteria carrying wild-type $h o p X_{P p h \text { race4 }}$ consistently grew 10 -fold less, due to $R 2$ function. Strains with mutations in each of the proposed catalytic triad residues avoided $R 2$-mediated disease resistance. The strain carrying the D233A allele grew to inter-

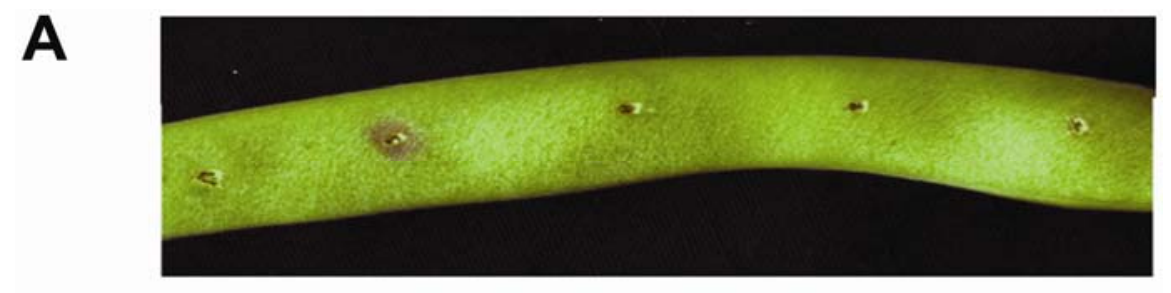

B
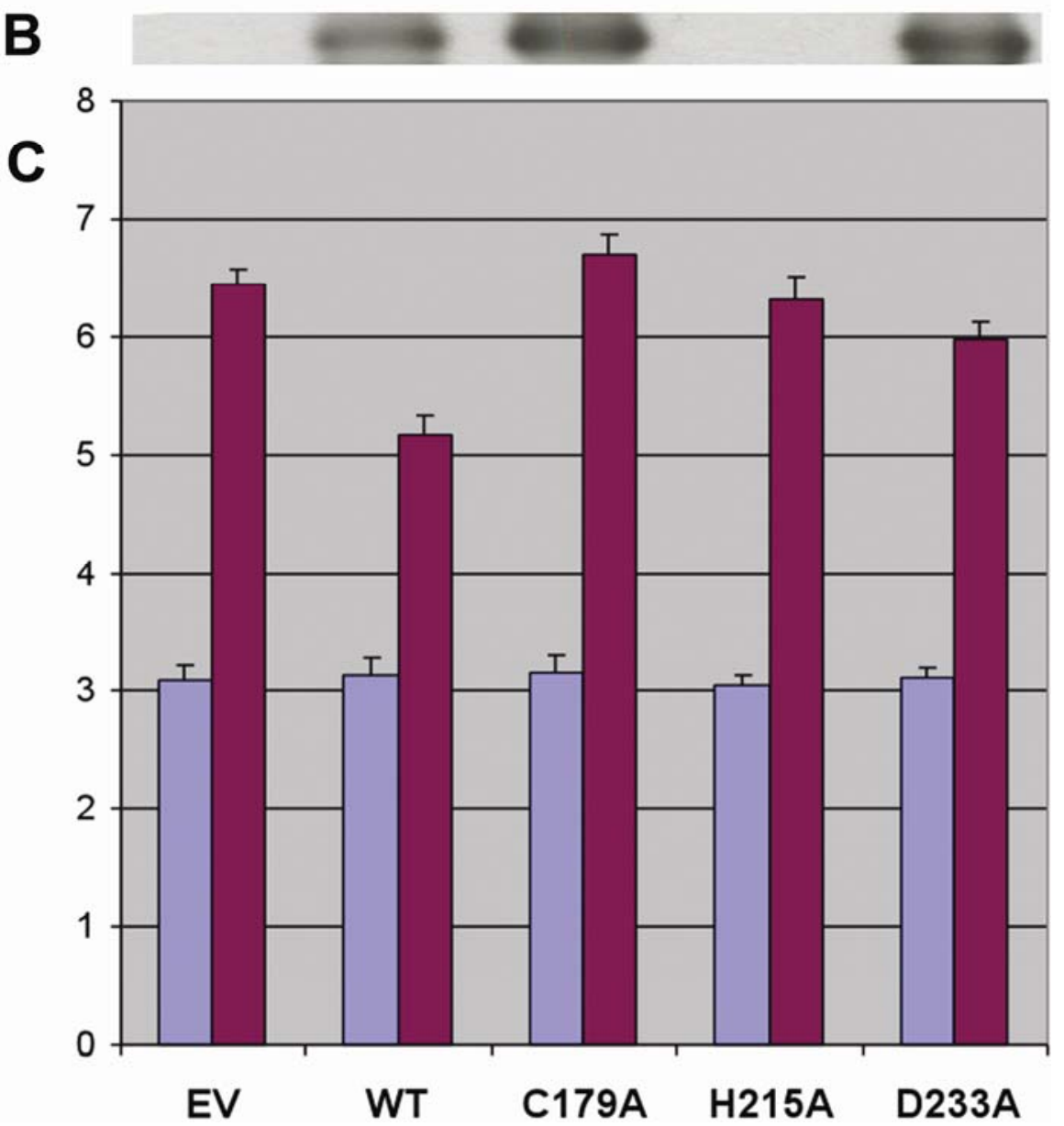

Fig. 2. The putative catalytic triad residues of $\mathrm{HopX}_{P p h \text { race } 4}$ are required for avirulence function in bean. The bean cultivar A43, containing the $R 2$ resistance gene, was inoculated with Pseudomonas syringae pv. phaseolicola race 6 containing vector alone (EV), wild-type hopX-HA$A_{P p h}$ race 4 (WT), or the hopX-HA $A_{P p h}$ race 4 catalytic triad mutants as indicated at the bottom. A, High-dose inoculation $\left(5 \times 10^{7} \mathrm{CFU} / \mathrm{ml}\right)$ hypersensitive response (HR) assays on a bean pod. HR sites were scored as browning around the initial inoculation site at $24 \mathrm{~h}$ postinoculation. Only WT (second from left) gave an HR. B, Western blot of HopX-HA derivatives expressed in P. syringae pv. phaseolicola race 6. C, Low-dose inoculation $\left(1 \times 10^{5} \mathrm{CFU} / \mathrm{ml}\right)$ and analysis of in planta bacterial growth at day 0 (blue bars), day 3 (red bars). Error bars represent standard deviation from triplicate samples. HR and bacterial growth assays were repeated three times with similar results. In each experiment, three separate pods were inoculated for HR assay. 
mediate levels in repeated experiments. We cannot accurately asses the importance of the $\mathrm{H} 215 \mathrm{~A}$ in $\mathrm{R} 2$-mediated disease resistance against HopX, since this protein does not accumulate to normal levels in bacterial cells (Fig. 2B and described below). However, it is clear that the putative catalytic Cys residue is necessary and that the Asp contributes towards the ability of HopX to activate R2-mediated disease resistance.

We also tested whether other HopX family members could trigger $R 2$ function. We tested alleles and putative catalytic Cys to Ala substitution mutant alleles from $P$. syringae pv. tomato $\mathrm{DC} 3000, P$. syringae pv. syringae $\mathrm{B} 728 \mathrm{a}$, and the avrXccE1 allele from X. campestris pv. campestris (Fig. 3). Like hop $X_{P p h \text { race4, }}$, both HopX $X_{P t o}$ DC3000 and HopX $\mathrm{X}_{P s y}$ B728a also triggered full $R 2$-mediated $\mathrm{HR}$ that was dependent on the proposed catalytic cysteine residue in each allele (Fig. 3A). The HR was triggered despite broad differences in protein accumulation of the respective wild-type alleles (Fig. 3B). HR assay results using these alleles on bean cultivars A43, Canadian Wonder, Tendergreen, and Red Mexican (Stevens et al. 1998) were identical to that of $h o p X_{P p h \text { race4 }}$, demonstrating that $R 2$ was responsible for recognition (data not shown). These data were confirmed by $R 2$-mediated restriction of pathogen growth (Fig. 3C). These results suggest that divergent alleles of hopX have maintained catalytic function and that putative catalytic residues are important for conserved avirulence functions recognized by the bean $R 2$ disease-resistance gene. In contrast, avrXccE1 triggered weak $R 2$ function on $\mathrm{A} 43$ bean, as evidenced by a smaller differential response to infection measured by either HR of pathogen growth comparing bacteria expressing an Ala substitution in the putative catalytic Cys or wild-type AvrXccE1 (Fig. 3A and C).

We next tested mutations in the other proposed catalytic residues of $\mathrm{HopX}_{P t o \text { DC3000 }}(\mathrm{H} 218 \mathrm{~A})$ and aspartic acid (D236A). Both of these mutations also abolished R2-dependent recognition (Fig. 4A). Relatively small amounts of wild-type protein (Fig. 4B) were sufficient to activate $R 2$ function and to restrict pathogen growth (Fig. 4C). Unlike the histidine mutation in $\mathrm{HopX}_{P p h \text { race4 }}$, HopX $\mathrm{X}_{\text {Pto DC3000 }}(\mathrm{H} 218 \mathrm{~A})$ was stable (Fig. 4B), al-
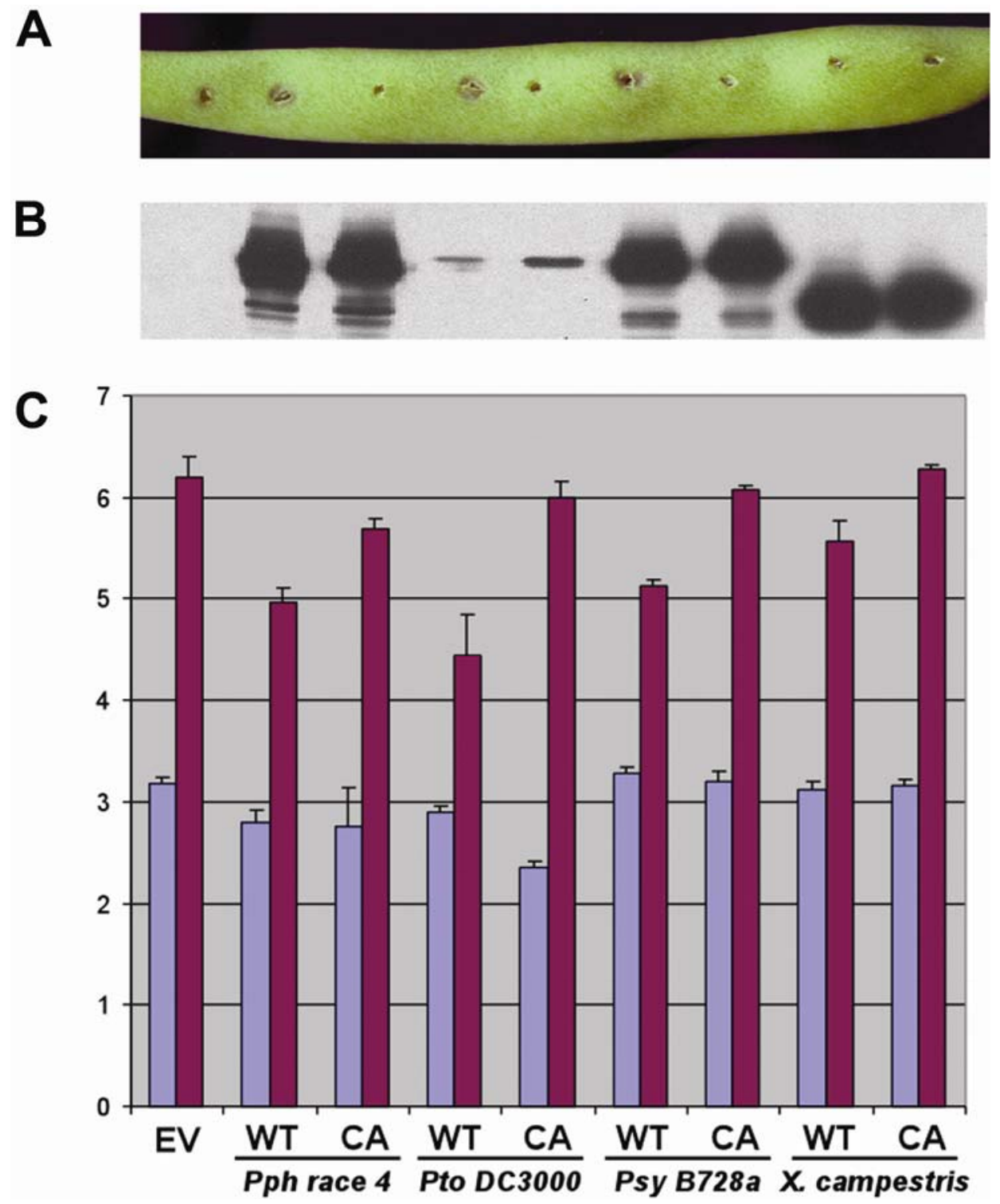

Fig. 3. The ability to trigger bean R2 is conserved among most HopX family members and requires the putative catalytic cysteine. Wild-type (WT) HopX, and Cys to Ala putative catalytic residue (CA) mutants of HopX alleles from each of the pathogens listed at bottom were tested in various assays. A, Hypersensitive response (HR) assays on the bean cultivar A43. B, Western blot of HopX-HA derivatives expressed in Pseudomonas syringae pv. phaseolicola race 6. C, Analysis of in planta bacterial growth on bean cultivar A43. Error bars represent standard deviation from triplicate samples. HR and bacterial growth assays were repeated three times with similar results. In each experiment, three separate pods were inoculated for HR assay. 
lowing us to conclude that this putative catalytic residue is critical for triggering the $R 2$ function. We noted in repeated experiments that HopX $\mathrm{X}_{P t o} \mathrm{DC} 3000$ mutants that abolished function were more stable than wild type (discussed below).

\section{HopX family members require putative catalytic residues in order to trigger a novel cell-death response on Arabidopsis.}

Direct expression of type III effectors in host cells can be used to identify relevant host targets (Grant et al. 2006; Mudgett 2005). In many animal bacteria systems, the expression of individual T3SS effectors directly in host cells can phenocopy aspects of infection in the absence of pathogen. Alternatively, overexpression of a given type III effector could result in activation of an NB-LRR normally not responsive to wild-type levels. Therefore, we conditionally expressed hop $X_{P t o}$ DC3000 in a variety of Arabidopsis ecotypes, using an Agrobacterium-mediated dexamethasone (DEX)-inducible transient expression system (Nimchuk et al. 2000). DEX- induced transient expression of hop $X_{P t o \text { DC3000 }}$ led to cell death in Col-0, Ws-0, and La-er accessions of Arabidopsis (Fig. 5A, La-er data not shown). In contrast, expression of hop $X_{P t o ~ D C 3000}$ did not elicit responses in accessions Bch-1 and Aa-0. Differences in phenotype were not due to differences in expression levels of HopX $\mathrm{X}_{\text {Pto DC3000 }}$ (Fig. 5B).

We determined the subcellular location of wild-type HopX $_{\text {Pto DC3000 }}$ inside host cells following conditional transient expression in Col-0 leaves (Fig. 5C). We determined that HopX $_{P t o \text { DC } 3000}$ was located in the soluble fraction, suggesting it acts on a target in the host cell cytosol. Crude fractions enriched for nuclei, chloroplasts, and mitochondria did not contain detectable levels of HopX $X_{\text {Pto DC3000 }}$ (data not shown).

We next addressed the role of the putative catalytic triad residues in the cell-death response of Arabidopsis. C-terminal HA-tagged versions of wild-type and mutant HopX $\mathrm{X}_{P t o}$ DC3000 were expressed transiently in Arabidopsis leaves. Transformation of Col-0 leaves with wild-type hopX $X_{P t o \text { DC3000 }}$ led to cell death $24 \mathrm{~h}$ after DEX induction, while vector alone controls
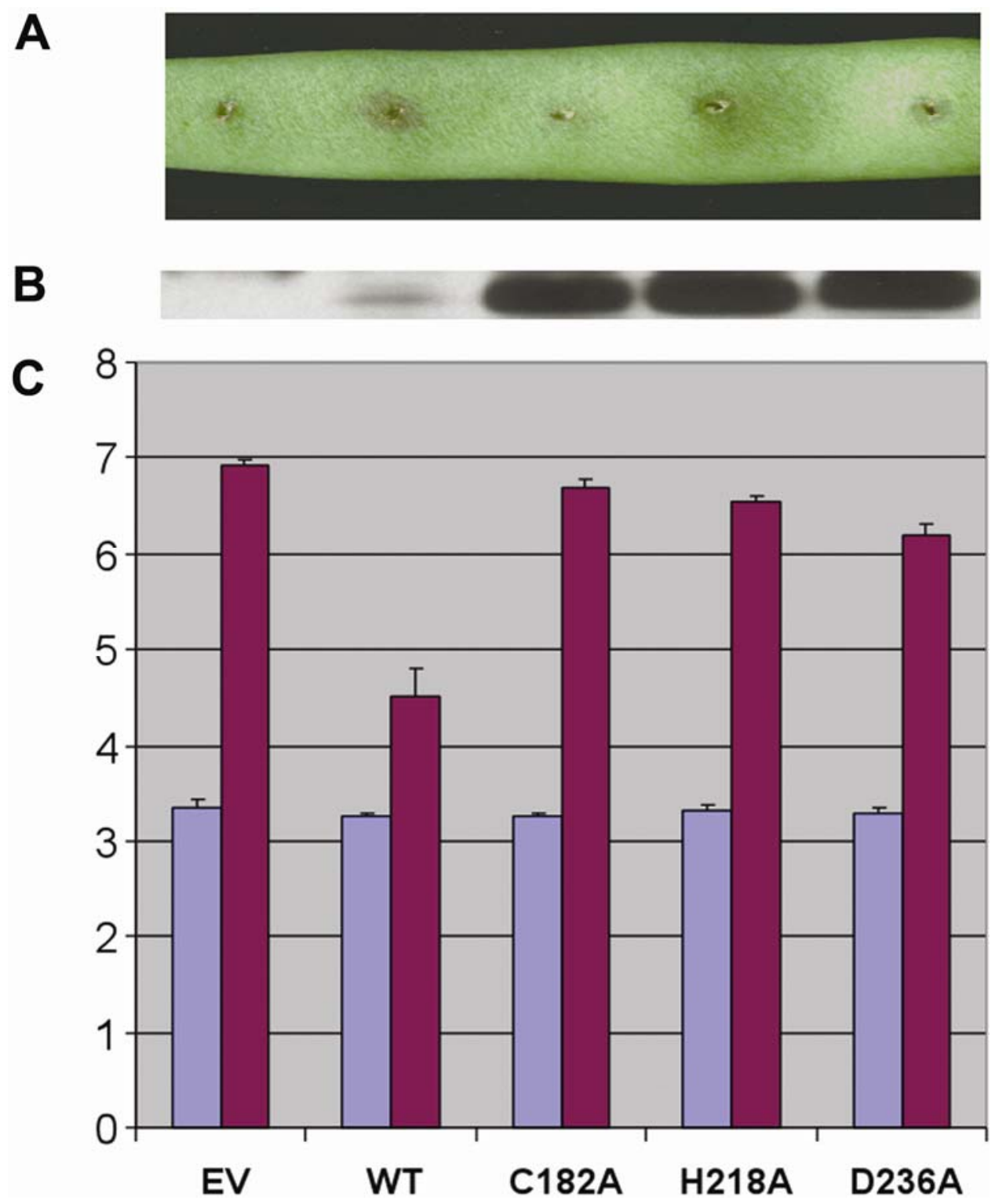

Fig. 4. The putative catalytic triad residues of HopX $\mathrm{X}_{P t o} \mathrm{DC} 3000$ are required for avirulence function in bean. The bean cultivar A43 was inoculated with $P s e u-$

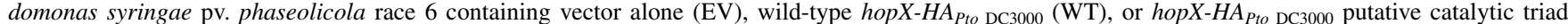

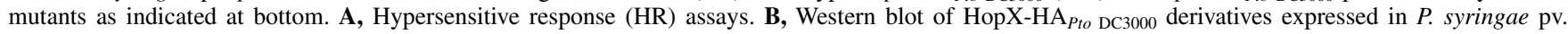
phaseolicola race 6. C, Analysis of in planta bacterial growth. Error bars represent standard deviation from triplicate samples. The experiment was repeated two times with similar results. In each experiment, three separate pods were inoculated for HR assay. 
did not (Fig. 6A). Mutations in the putative catalytic sites (C182A, H218A, and D236A) abolished this response, yet these mutations did not negatively affect protein accumulation (Fig. 6B).

We next asked whether other hopX alleles could trigger host cell death in Arabidopsis. We utilized the Ws-0 accession, as we found it more amenable to Agrobacterium-mediated transient expression (not shown). Both HopX $\mathrm{X}_{P t o}$ DC3000 and HopX $\mathrm{X}_{P S \mathrm{y}}$ ${ }_{\mathrm{B} 728 \mathrm{a}}$ triggered a cell-death response that was dependent on the putative catalytic cysteine residues of each (C182A or C182S and $\mathrm{C} 183 \mathrm{~A}$, respectively), while $\mathrm{HopX}_{P p h \text { race4 }}$ and AvrXccE1 did not trigger the cell-death response (Fig. 7A). Again, protein levels were not compromised by these mutations (Fig. 7B). Thus, the ability to trigger this phenotype on Arabidopsis is polymorphic across the HopX family, and the conserved putative catalytic residues are required for it among the active alleles. We envision that this phenotype reflects an interaction with a potential target in Arabidopsis.

It is interesting to note that AvrXccE1, which initiates only very weak $R 2$ function on bean and no cell death in Arabidop- sis, contains an $\mathrm{N}$-terminal consensus eukaryotic $\mathrm{N}$-myristoylation sequence previously shown to drive effectors to the host plasma membrane (Nimchuk et al. 2000; Robert-Seilaniantz et al. 2006; Shan et al. 2000). It is possible that the lack of plant response to AvrXccE1 is due to its localization to the plasma membrane.

We were unable to detect a difference in bacterial virulence due to the presence or absence of hopX on either susceptible bean cultivar using $P$. syringae pv. phaseolicola race 6 , or in Arabidopsis using $P$. syringae pv. tomato DC3000, or P. syringae pv. maculicola race 6 (data not shown) using combinations of deletion mutants for hopX and hopX overexpressing plasmids. We were also unable to replicate the ability of HopX to block HopPsyA-triggered HR on the Arabidopsis Ws- 0 accession when delivered from $P$. fluorescens (Jamir et al. 2004).

\section{Conserved domain $\mathrm{A}$ is required}

for HopX function in bean and Arabidopsis.

We tested the necessity of conserved residues in domain A for both HopX-associated phenotypes, triggering of R2 func-

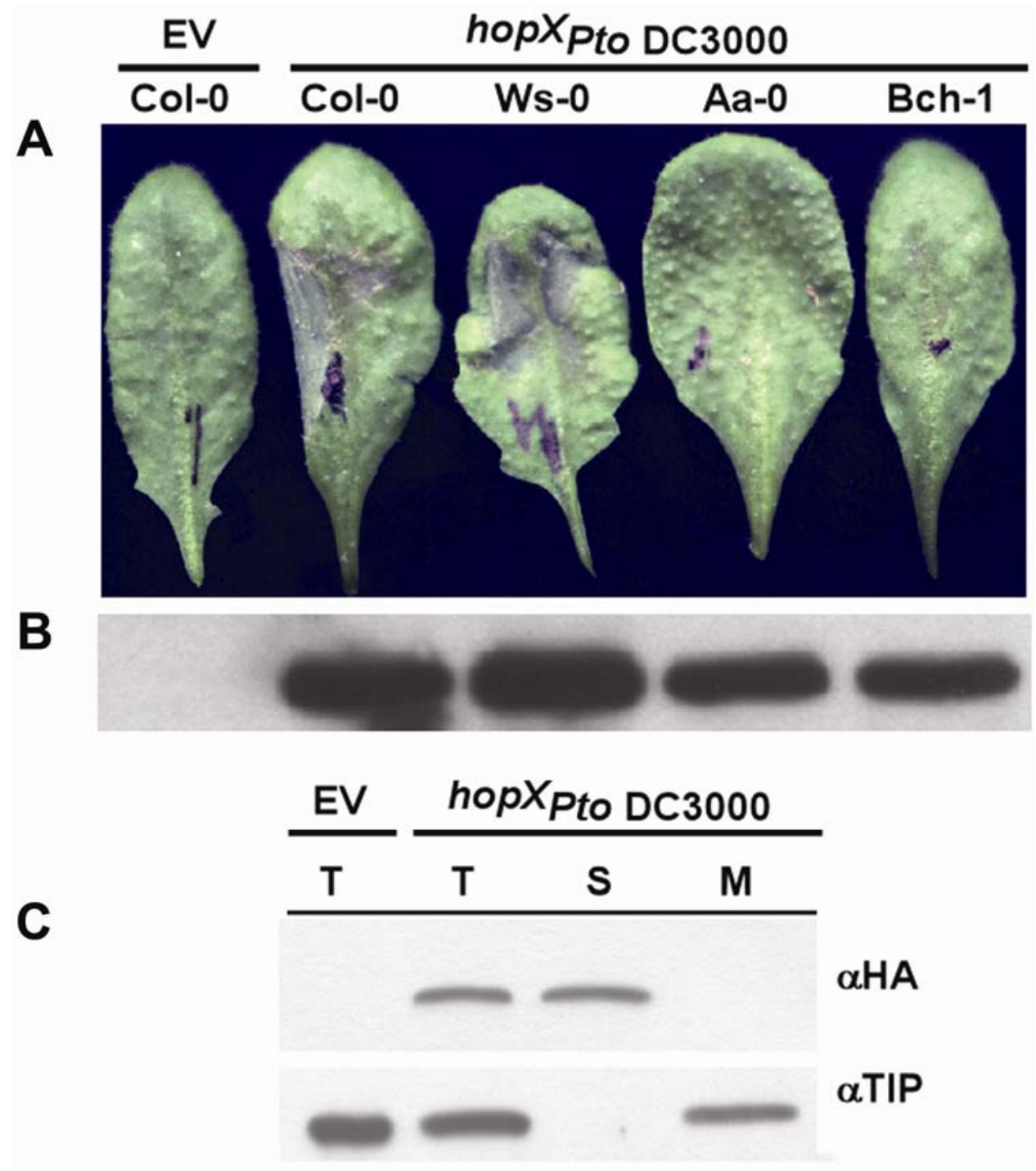

Fig. 5. HopX $\mathrm{X}_{\text {Pto DC3000 }}$ triggers genotype-dependent host cell death when expressed in Arabidopsis and is soluble. Arabidopsis leaves were inoculated with Agrobacterium cells with a T-DNA carrying $h o p X_{P t o}$ DC3000 and were subsequently sprayed with dexamethasone (DEX). A, Arabidopsis accessions (left to right): Col-0 (empty vector control), Col-0, Ws-0, Aa-0, and Bch-1. Photos taken $24 \mathrm{~h}$ after DEX treatment. B, Anti-HA Western blot from transiently transformed plants $12 \mathrm{~h}$ after DEX treatment; $20 \mu \mathrm{g}$ of total protein were loaded. C, Crude cellular fractionation of HopX-HA. T = total, $\mathrm{S}=$ soluble, $\mathrm{M}=$ microsomal fractions, and TIP = tonoplast intrinsic protein for membrane fractionation control. Equivalent fraction volumes were loaded. 16 leaves from four individual plants were inoculated per treatment. Similar results were obtained in four experiments. 
tion on bean and cell death in Arabidopsis. Using hopX $X_{P t o}$ DC3000, we generated a triple alanine substitution in this region, corresponding to residues R122, N124, and D126. This allele failed to trigger $R 2$ function but did not decrease protein accumulation levels when expressed in $P$. syringae pv. phaseolicola race 6 (Fig. 8A, 8, and C). In addition, the triple alanine substitution did not trigger cell death in Arabidopsis Ws-0 plants when expressed via Agrobacterium transient transformation, even though it accumulated to high levels in planta (Fig. 8D and E). Thus, the conserved domain $\mathrm{A}$ is also required for HopX function in bean and Arabidopsis.

\section{DISCUSSION}

We report that the HopX family joins a growing list of T3SS effectors from phytopathogenic bacteria that share homology with enzymes. While we were unable to detect in vitro enzyme activity, bioinformatics and genetics strongly suggest that the HopX family encode active enzymes of the TGase catalytic triad superfamily. HopX family members contain a conserved domain highly similar in sequence and secondary structure to the catalytic triads of TGase superfamily members. There is particular homology between certain HopX alleles and the catalytic triad of eukaryotic PNGases. Mutation in the residues of this putative catalytic triad of various HopX family members abolished avirulence activity on $R 2$-expressing bean cultivars and also prevented initiation of cell death in Arabidopsis following transient conditional expression assays. These data suggest that HopX family members are putative enzymes and that this activity is required for both $R$-mediated recognition and for potential virulence functions in susceptible hosts.

The lack of demonstrable enzyme activity prevents us from discounting the possibility that the conserved triad-like domain in HopX functions in a structural, rather than enzymatic, man-

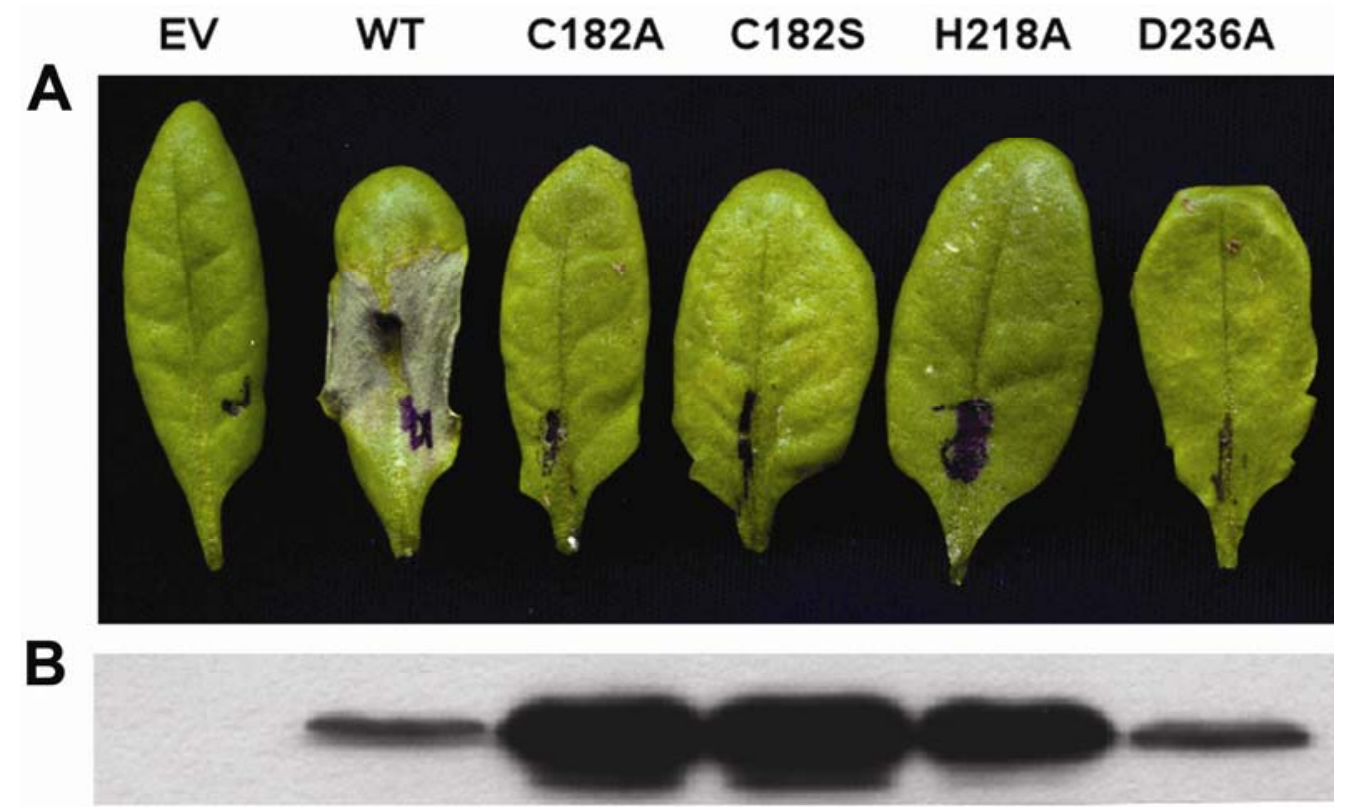

Fig. 6. The putative catalytic triad of HopX $\mathrm{X}_{P t o} \mathrm{DC} 3000$ is required to initiate Arabidopsis cell death. A, Col-0 (rpml-3) plants were inoculated with Agrobacterium cells carrying a T-DNA with hopX-HA $A_{P t o}$ DC3000 or proposed catalytic triad mutant alleles as listed at the top. B, Anti-HA Western blot from plant transiently transformed plants $12 \mathrm{~h}$ after dexamethasone treatment; $20 \mu \mathrm{g}$ of total protein were loaded. A total of 16 leaves from four individual plants were inoculated per treatment. Experiments were repeated with similar results three times.

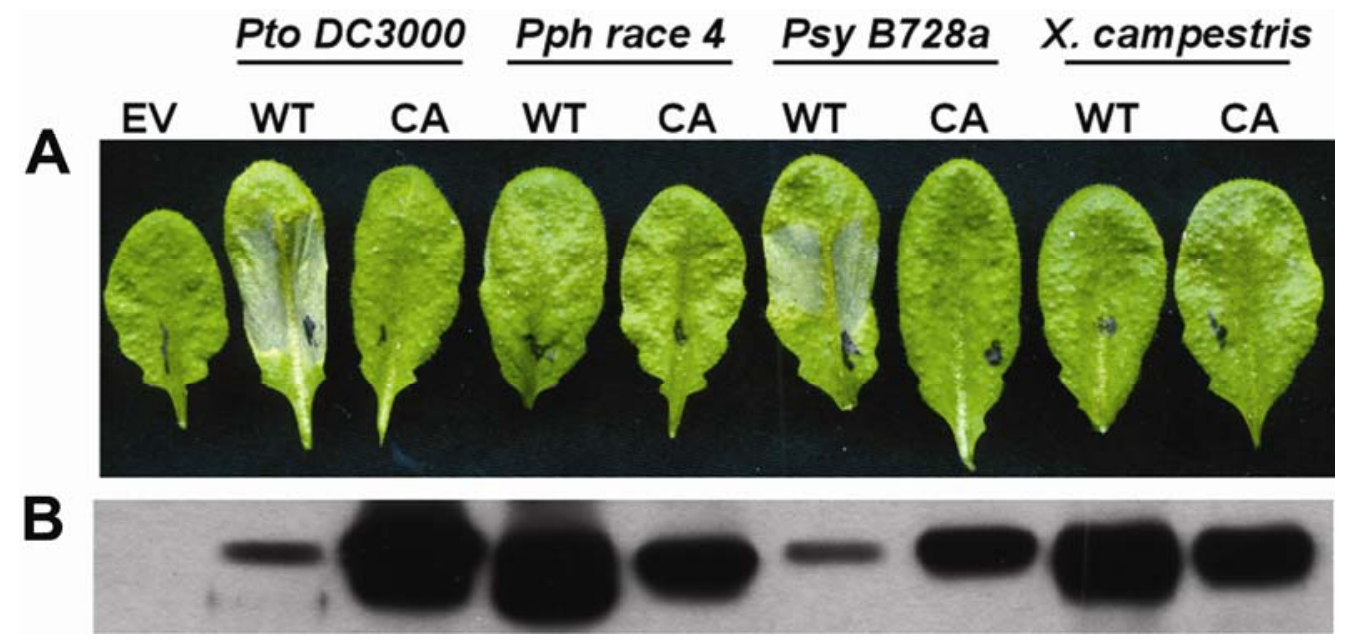

Fig. 7. The ability to trigger Arabidopsis cell death differs among HopX family members. A, Inoculated Ws- 0 plants. WT $=$ wild-type HopX. CA $=$ putative active site cysteine to alanine mutant of the HopX allele from each of the pathogens listed at the top. B, Anti-HA Western blot from transiently transformed plants, $12 \mathrm{~h}$ after dexamethasone treatment; $20 \mu \mathrm{g}$ total protein were loaded. A total of 16 leaves from four individual plants were inoculated per treatment. Experiments were repeated with similar results three times. 
ner. However, we find this unlikely, given the overall considerable sequence variability in the HopX family and the precedence of catalytic triads occurring in other type III effectors. Other $P$. syringae type III effectors, including AvrRpt2 AvrPphB, and HopPtoN, are cysteine proteases in classes distinct from the TGase superfamily (Axtell et al. 2003; LopezSolanilla et al. 2004; Shao et al. 2002). AvrRpt2, for example, undergoes host cyclophillin-dependent autoprocessing in planta to release the active catalytic domain (Coaker et al. 2005). We have not detected a similar autoprocessing for HopX in either plant or bacterial extracts (data not shown). The catalytic domain of HopX is most similar to that of peptide N-glycanases, however, there does not appear to be any conserved homology outside of the catalytic triad, including no clear PUB domain or zinc-coordinating center found in eukaryotic PNGases (Lee et al. 2005; Suzuki et al. 2002). Given the highly selective substrate requirements for most enzymatic T3SS effectors characterized to date, assignment of a precise enzyme function to HopX will most likely be determined only when the host target in known.

Iterative blast search revealed many proteins that contained weak homology to the HopX catalytic triad (data not shown). However, the only protein identified that contained similarity to both domain A and the putative catalytic triad of HopX was the Lpg2408 protein from L. pneumophila. It is tempting to speculate that this protein might act as an effector of the DOT/ICM secretion system in this animal pathogen. Effector proteins of the DOT/ICM system in L. pneumophila contain

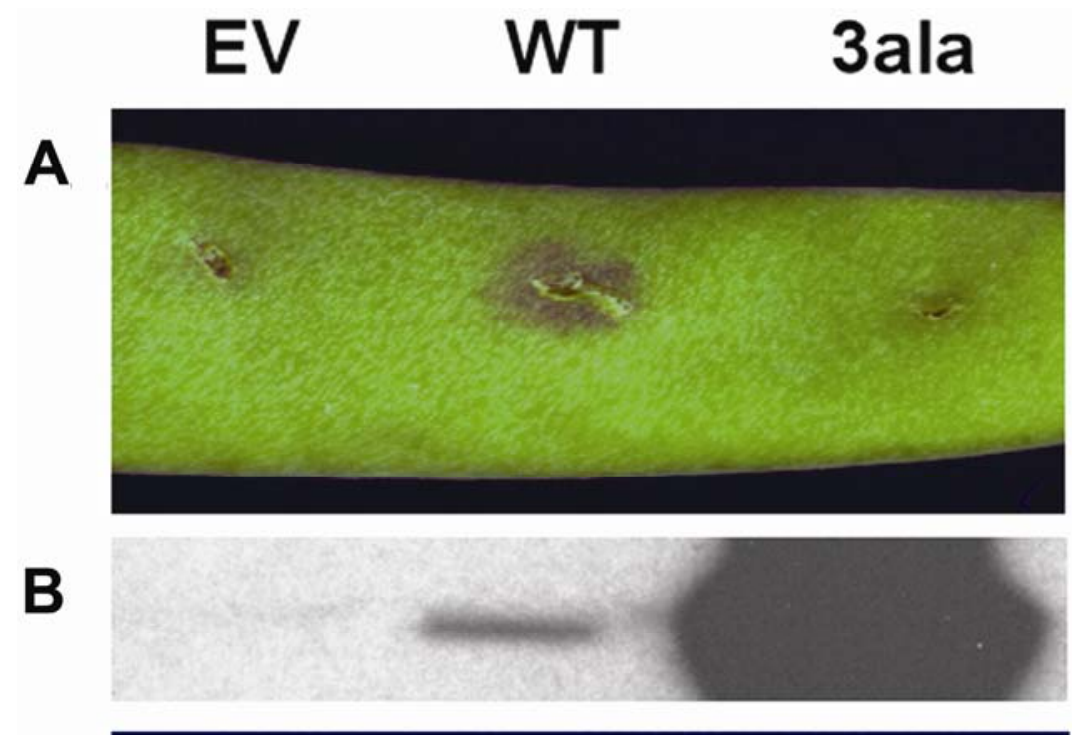

\section{C}
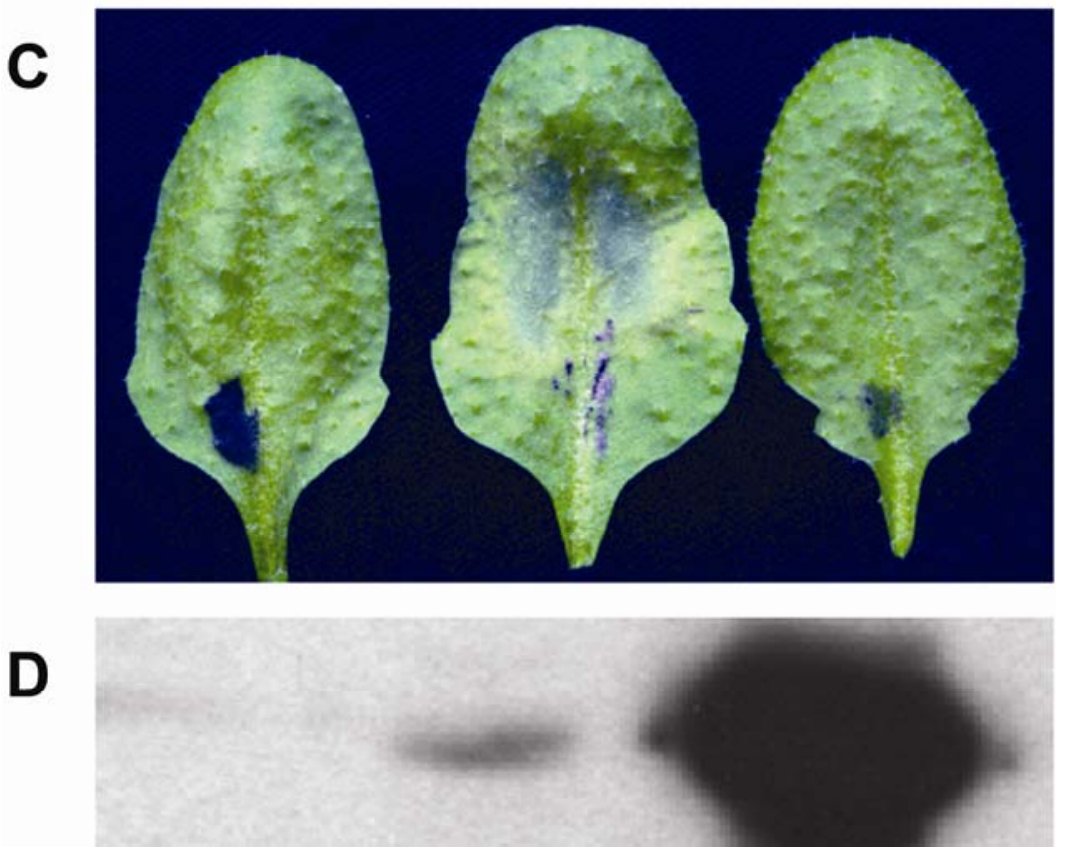

Fig. 8. The conserved domain A is also required for HopX functions in both bean and Arabidopsis. A, Bean high-dose hypersensitive response (HR) assays. Bean cultivar A43 were inoculated with Pseudomonas syringae pv. phaseolicola race 6 containing vector alone (EV), wild-type hopX-HA $A_{P t o}$ DC3000 $(\mathrm{WT})$ or

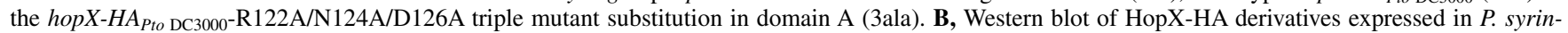
gae pv. phaseolicola race 6. C, HopX-HA ${ }_{P t o}$ DC3000 growth curve on bean cultivar A43. Error bars represent standard deviation from triplicate samples. Experiments were repeated two times with similar results. D, Arabidopsis cell-death assay performed on Ws-0, using Agrobacterium transferring vector alone (EV), wild-type $h o p X-H A_{P t o}$ DC3000 $(W T)$, or the $h o p X-H A_{P t o}$ DC3000-R122A/N124A/D126A triple mutant substitution in domain A (3ala). E, Anti-HA Western blot from transiently transformed plants $12 \mathrm{~h}$ after dexamethasone treatment; $20 \mu \mathrm{g}$ of total protein were loaded. A totala of 16 leaves from four individual plants were inoculated per treatment. Experiment was repeated with similar results three times. In each experiment, three separate pods were inoculated for HR assay. 
hydrophobic residues at the -3 and -4 positions in their $\mathrm{C}$-terminus (Nagai et al. 2005). The Lpg2408 C-terminus has leucine and proline residue at the -3 and -4 positions, respectively. The observation that Lpg2408 has a domain A suggests that this is not a T3SS chaperone-binding domain in HopX, as L. pneumophila lacks a T3SS secretion system (Chien et al. 2004). The Lpg2408 protein also lacks the N-terminal extension upstream of domain A observed in the HopX family, which is not surprising as this domain in T3SS effectors is necessary and sufficient for translocation via the T3SS (Schechter et al. 2004; Sorg et al. 2005). Thus, while Lpg2408 has not been reported as a DOT/ICM substrate protein, the homology to HopX and the presence of a potential DOT/ICM translocation signals at the C-terminus suggest that the HopX family is not restricted to plant-pathogenic bacteria. Conservation of T3SS effector proteases between animal and plant pathogens has been noted previously; however, it is not clear if these have a conserved host target (Orth et al. 2000; Shao et al. 2002). If HopX and Lpg2408 are both deployed as effectors, it would suggest that they and perhaps other virulence factors can be substrates for either the T3SS or type IV secretion systems through the addition or deletion of appropriate $\mathrm{N}$ - or C-terminal secretion signals. This would have implications for how effectors evolve over time and are dispersed throughout bacteria (Hubber et al. 2004).

Secondary structure prediction suggests that domain A may form a helix-loop-helix with the most conserved GXXN motif in the putative loop region (Fig. 1C). The homology between Lpg2408 and HopX included residues within the A domain, including the conserved glycine (Gly121 in HopX $\mathrm{X}_{\text {Pto DC3000). In }}$ $\operatorname{Lpg} 2408$, this region is also predicted by secondary structure programs to be in a loop between two helices as well (data not shown). This subdomain has weak homology to the coenzymebinding Rossman fold of some dehydrogenase/reductases, though it is not predicted to form a glycine-rich Rossman fold found in nucleotide binding domains (data not shown). The predicted helical arrangement in domain $\mathrm{A}$ is also reminiscent of the helix-loop-helix motif utilized by C3-exotoxin (C3bot) from Clostridium botulinum to bind its host target RalA. Mutations in the loop residues in $\mathrm{C} 3 \mathrm{~B}$ ot abolish interaction with RalA (Pautsch et al. 2005). The helix-loop-helix motif is also found in $\mathrm{Ca} 2^{+}$-binding motifs of eukaryotic EF hands and prokaryote $\mathrm{EF}$ variants; however, conserved aspartic acid residues that are present in the loop domain of EF hands are not present in the loop of domain A in HopX (Kawasaki et al. 1998). We hypothesize that domain A may represent either a host-target interaction domain or a novel nucleotide/cofactor binding domain.

The ability to trigger $R$-mediated disease resistance is correlated with activity for all of the T3SS effectors for which enzyme function can be demonstrated or implied (Dangl and Jones 2001; Nimchuk et al. 2001). In at least two cases, proteolytic cleavage of a host target protein by an effector is required for activation of the corresponding NB-LRR protein (Axtell and Staskawicz 2003; Mackey et al. 2003; Shao et al. 2003). We noted above that the HopX alleles from $P$. syringae pv. tabaci or HopX $\mathrm{X}_{P p h \text { race6 }}$ could not trigger $R 2$ function in bean, although they both possess an intact putative catalytic triad and, thus, could be functional enzymes (Stevens et al. 1998). Stevens and associates (1998) also noted that $R 2$-inactive HopX $\mathrm{X}_{P h \text { racel }}$ contains a single G191R substitution compared with the $R 2$-active $\mathrm{HopX}_{P p h \text { race4 }}$. This residue is just between the catalytic cysteine and histidine and could alter catalytic function. Further, just C-terminal to the catalytic aspartic acid is invariant tryptophan 235 in the R2-active $\mathrm{HopX}_{P p h \text { race4, which is }}$ changed to leucine in the $R 2$-inactive $\mathrm{HopX}_{P p h \text { raceg. }}$ The aminoacid differences between the $R 2$-inactive $\mathrm{HopX}_{P p h}$ racel and

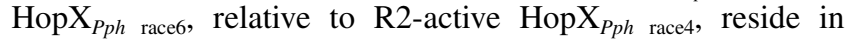

different domains of the protein. Thus, while putative catalytic residues are necessary for avirulence function, additional residues are also required. Similarly, all HopX alleles share the conserved domain A. Therefore, additional residues outside of this domain must contribute to avirulence function. The region C-terminal to the catalytic domain of the HopX family is much more variable among different phytopathogenic bacterial species and, thus, might be a region that determines differential target specificity (data not shown). Consistent with this, the $R 2$ inactive $\mathrm{HopX}_{P p h \text { race6 }}$ differs from the $R 2$-active HopX $\mathrm{X}_{P h \text { race4}}$, $\mathrm{HopX}_{P t o \text { DC3000, and HopX }} \mathrm{X}_{P y \text { B728a }}$ proteins by only an E310K substitution in this region.

Taken together with our data, we suggest i) HopX has homology to TGase superfamily enzymes, ii) putative enzyme activity is necessary but not sufficient for HopX avirulence function; and iii) HopX may contact host targets involved in triggering R2 function using multiple domains. Because HopX presumably did not evolve to trigger R2 function but rather for some as-yet-undefined virulence function, we favor the idea that R2 is not the direct target of HopX enzyme activity but acts as a coreceptor.

HopX is sufficient to trigger host cell death when expressed in Arabidopsis. In animal models of T3SS pathogen interactions, the expression of individual T3SS effectors in host cells has proven to be a reliable way to annotate T3SS effector function and to identify relevant host targets for over 15 effectors (Hueck 1998; Kjemtrup et al. 2000). We believe that the cell death induced by conditional expression of $\mathrm{HopX}_{P t o} \mathrm{DC} 3000$ in Arabidopsis may reflect an interaction of $\mathrm{HopX}_{P t o}$ DC3000 with and modification of a relevant host target. The nature of the cell-death response is unknown. It could reflect sustained interference with host signaling system necessary for basic cell functions or it may be due to activation of an unknown Arabidopsis $\mathrm{R}$ protein. The polymorphic nature of the response among Arabidopsis ecotypes is more consistent with $\mathrm{R}$ protein activation; however, a few observations suggest otherwise. First, hop $X_{\text {Pto DC3000-dependent cell death was not compromised }}$ following Agrobacterium-mediated transient expression assays on a diverse array of Col-0-derived Arabidopsis mutants that alter or abolish NB-LRR-mediated or basal defense responses (data not shown). Second, HopX $\mathrm{X}_{P t o}$ DC3000 does not normally trigger an $R$-dependent response on Arabidopsis, and $P$. syringae pv. tomato DC3000 carrying this gene is an aggressive pathogen. If Hop $\mathrm{X}_{P t o \text { DC } 3000^{-}}$triggered cell death is due to R protein activation, it would imply that this response is masked during $P$. syringae pv. tomato DC3000 infection. Ultimately, identification of the host target in Arabidopsis that is responsible for AvrPphE cell death will clarify the nature of this response, and the data presented here will focus attention on the relevant regions of the HopX proteins. To date, direct targets of T3SS effectors have been identified initially through R-mediated responses, using either genetic or biochemical methods (Mackey et al. 2002; Warren et al. 1999). We envision that direct targets for HopX may be identified by using the Arabidopsis genetic model even if the cell-death response is due to $\mathrm{R}$ protein activation.

\section{MATERIALS AND METHODS}

\section{Bioinfomatics.}

The translated HopX $\mathrm{X}_{P h \text { race4 }}$ sequence was used in the PSIBLAST program to query GenBank and identify putative homologs. HopX family members were imported into Vector NTI and were aligned using the Align-X function of the program. Iterative PSI-BLASTs were used to find distantly related homologs, using either the full amino-acid sequence of $\mathrm{HopX}_{P p h \text { race4 }}$ or the A domain or catalytic triad region alone. 
Secondary structure predictions for HopX proteins were generated using the JPRED (Cuff et al. 1998) consensus program. It was noted that this program effectively predicted the T3SS effector AvrB secondary structure prior to publishing (data not shown).

\section{$P$. syringae HR and in planta growth assays on bean.}

P. syringae pv. phaseolicola race 6 (gift from J. Mansfield) strains were grown and inoculated in plants as described by Debener and associates (1991) and Mansfield and associates (1994). P. syringae were resuspended to an optical density at $600 \mathrm{~nm}=0.1$ (corresponding to $5 \times 10^{7} \mathrm{CFU} / \mathrm{ml}$ ) for $\mathrm{HR}$ assays on bean pods or $1 \times 10^{5} \mathrm{CFU} / \mathrm{ml}$ for growth curves on bean leaves. HR was scored from $20 \mathrm{~h}$ for hopX-R2. Bean cultivars A43, Canadian Wonder, Tendergreen and Red Mexican were grown according to Mansfield and associates (1994).

\section{$\boldsymbol{P}$ syringae protein extraction and analysis.}

P. syringae cultures $(2.0 \mathrm{ml})$ were grown overnight in King's B media with appropriate antibiotics. Cells $\left(5 \times 10^{7}\right)$ were pelleted and resuspended in $200 \mu \mathrm{l}$ of $1 \times$ Laemmli buffer (Laemmli 1970) and were boiled at $90^{\circ} \mathrm{C}$ for $2 \mathrm{~min}$. Samples (5 $\mu \mathrm{l} \mathrm{each)}$ was loaded on $10 \%$ sodium dodecyl sulfate-polyacrylamide gel electrophoresis gels and were transferred via Western blotting. HA-tagged proteins were detected using a highaffinity monoclonal HA antibody (Roche, Branchburg, NJ, U.S.A.) with a Rat polyclonal secondary (Sigma, St. Louis). Equal loading of gels was monitored by Coomassie brilliant blue- and Ponceau S-staining of gels and membranes, respectively.

\section{Construction of clones and mutants.}

All hopX alleles were PCR amplified from genomic DNA of their corresponding strains, using Pfu polymerase (Stratagene, La Jolla, CA, U.S.A.). Forward primers added Xho 1 and Nde 1 sites before the ATG. Reverse primers added the HA epitopetag sequence followed by a stop codon and a Spe 1 site. Primer sequences are available on request. PCR products were cloned into TOPO pCR2.1 (Invitrogen, Carlsbad, CA, U.S.A.) and were sequenced. For expression in $P$. syringe, hopX alleles were cloned as Nde1-Spe1 fragments behind a dual lac-nptII promoter in a modified pCR2.1 vector (unpublished) and were then cloned into the broad host range vector pBR1-MCS2 (Kovach et al. 1995) as either an Xho1-Spe1 fragment or either an EcoR1 fragment, BamH1 fragment, or both. Agrobacterium sp.-based in planta expression constructs were generated by cloning Xho1-Spe1 from pCR2.1 clones directly into a modified version of the pTA7002 vector (McNellis et al. 1998) containing the Basta herbicide resistance gene.

\section{Agrobacterium DEX-inducible transient expression assays.}

Transient transformation assays were performed, as previously described, using $20 \mu \mathrm{M}$ DEX (Nimchuk et al. 2000) Arabidopsis were scored for phenotypes $20 \mathrm{~h}$ postinduction. Protein was extracted $8 \mathrm{~h}$ postinduction from four transformed leaf disks for Western blots as described above.

\section{Total plant extract fractionation.}

Plant cell fractionation and analysis was performed as previously described (Nimchuk et al. 2000).

\section{ACKNOWLEDGMENTS}

We thank J. Shock for her assistance. We also thank J. Mansfield, Imperial College, Wye, U.K., for providing $P$. syringae phaseolicola strains and bean seeds. This work was supported by grants from the National Institutes of Health (NIH) (RO1-GM-066025) and the United States Department of Energy (DE-FG05-95ER20187) to J. L. Dangl. D. Desveaux was a fellow of the Natural Science and Engineering Research Council (NSERC) of Canada. J. H. Chang was an NIH-National Research Service Award fellow (F32-GM20296-02S1).

\section{LITERATURE CITED}

Altschul, S. F., Madden, T. L., Schaffer, A. A., Zhang, J., Zhang, Z., Miller, W., and Lipman, D. J. 1997. Gapped BLAST and PSI-BLAST: A new generation of protein database search programs. Nucleic Acids Res. 25:3389-3402.

Axtell, M. J., and Staskawicz, B. J. 2003. Initiation of RPS2-specified disease resistance in Arabidopsis is coupled to the AvrRpt2-directed elimination of RIN4. Cell 112:369-377.

Axtell, M. J., Chisholm, S. T., Dahlbeck, D., and Staskawicz, B. J. 2003. Genetic and molecular evidence that the Pseudomonas syringae type III effector protein AvrRpt2 is a cysteine protease. Mol. Microbiol. 49:1537-1546.

Belkhadir, Y., Nimchuk, Z., Hubert, D. A., Mackey, D., and Dangl, J. L. 2004a. Arabidopsis RIN4 negatively regulates disease resistance mediated by RPS 2 and RPM1 downstream or independent of the NDR1 signal modulator and is not required for the virulence functions of bacterial type III effectors AvrRpt2 or AvrRpm1. Plant Cell 16:2822-2835.

Belkhadir, Y., Subramaniam, R., and Dangl, J. L. 2004b. Plant disease resistance protein signaling: NBS-LRR proteins and their partners. Curr. Opin. Plant Biol. 7:391-399.

Buell, C. R., Joardar, V., Lindeberg, M., Selengut, J., Paulsen, I. T., Gwinn, M. L., Dodson, R. J., Deboy, R. T., Durkin, A. S., Kolonay, J. F., et al. 2003. The complete genome sequence of the Arabidopsis and tomato pathogen Pseudomonas syringae pv. tomato DC3000. Proc. Natl. Acad. Sci. U.S.A. 100:10181-10186.

Charity, J. C., Pak, K., Delwiche, C. F., and Hutcheson, S. W. 2003. Novel exchangeable effector loci associated with the Pseudomonas syringae hrp pathogenicity island: Evidence for integron-like assembly from transposed gene cassettes. Mol. Plant-Microbe Interact. 16:495-507.

Chien, M., Morozova, I., Shi, S., Sheng, H., Chen, J., Gomez, S. M., Asamani, G., Hill, K., Nuara, J., Feder, M., et al. 2004. The genomic sequence of the accidental pathogen Legionella pneumophila. Science 305:1966-1968.

Chisholm, S. T., Coaker, G., Day, B., and Staskawicz, B. J. 2006. Host-microbe interactions: Shaping the evolution of the plant immune response. Cell 124:803-814.

Cianciotto, N. P. 2001. Pathogenicity of Legionella pneumophila. Int. J. Med. Microbiol. 291:331-343.

Coaker, G., Falick, A., and Staskawicz, B. 2005. Activation of a phytopathogenic bacterial effector protein by a eukaryotic cyclophilin. Science 308:548-550

Cuff, J. A., Clamp, M. E., Siddiqui, A. S., Finlay, M., and Barton, G. J. 1998. JPred: A consensus secondary structure prediction server. Bioinformatics 14:892-893.

da Silva, A. C., Ferro, J. A., Reinach, F. C., Farah, C. S., Furlan, L. R., Quaggio, R. B., Monteiro-Vitorello, C. B., Van Sluys, M. A., Almeida, N. F., Alves, L. M., et al. 2002. Comparison of the genomes of two Xanthomonas pathogens with differing host specificities. Nature 417:459-463.

Dangl, J. L., and Jones, J. D. 2001. Plant pathogens and integrated defence responses to infection. Nature 411:826-833.

Debener, T., Lehnackers, H., Arnold, M., and Dangl, J. L. 1991. Identification and molecular mapping of a single Arabidopsis thaliana locus determining resistance to a phytopathogenic Pseudomonas syringae isolate. Plant J. 1:289-302.

Deng, W. L., Rehm, A. H., Charkowski, A. O., Rojas, C. M., and Collmer, A. 2003. Pseudomonas syringae exchangeable effector loci: Sequence diversity in representative pathovars and virulence function in P. syringae pv. syringae B728a. J. Bacteriol. 185:2592-2602.

Desveaux, D., Singer, A. U., and Dangl, J. L. 2006. Type III effector proteins: Doppelgangers of bacterial virulence. Curr. Opin. Plant Biol. 9:376-382.

Dodds, P. N., Lawrence, G. J., Catanzariti, A. M., Teh, T., Wang, C. I., Ayliffe, M. A., Kobe, B., and Ellis, J. G. 2006. Direct protein interaction underlies gene-for-gene specificity and coevolution of the flax resistance genes and flax rust avirulence genes. Proc. Natl. Acad. Sci. U.S.A. 103:8888-8893.

Grant, S. R., Fisher, E. J., Chang, J. H., Mole, B. M., and Dangl, J. L. 2006. Subterfuge and manipulation: Type III effector proteins of phytopathogenic bacteria. Annu. Rev. Microbiol. 60:425-429.

He, P., Shan, L., Lin, N. C., Martin, G. B., Kemmerling, B., Nurnberger, T., and Sheen, J. 2006. Specific bacterial suppressors of MAMP signaling upstream of MAPKKK in Arabidopsis innate immunity. Cell $125: 563-575$ 
Hirsch, C., Blom, D., and Ploegh, H. L. 2003. A role for N-glycanase in the cytosolic turnover of glycoproteins. EMBO (Eur. Mol. Biol. Organ.) J. 22:1036-1046.

Hubber, A., Vergunst, A. C., Sullivan, J. T., Hooykaas, P. J., and Ronson, C. W. 2004. Symbiotic phenotypes and translocated effector proteins of the Mesorhizobium loti strain R7A VirB/D4 type IV secretion system. Mol. Microbiol. 54:61-574.

Hueck, C. J. 1998. Type III protein secretion systems in bacterial pathogens of animals and plants. Microbiol. Mol. Biol. Rev. 62:379-433.

Jamir, Y., Guo, M., Oh, H. S., Petnicki-Ocwieja, T., Chen, S., Tang, X., Dickman, M. B., Collmer, A., and Alfano, J. R. 2004. Identification of Pseudomonas syringae type III effectors that can suppress programmed cell death in plants and yeast. Plant J. 37:554-565.

Kawasaki, H., Nakayama, S., and Kretsinger, R. H. 1998. Classification and evolution of EF-hand proteins. Biometals 11:277-295.

Kjemtrup, S., Nimchuk, Z., and Dangl, J. L. 2000. Effector proteins of phytopathogenic bacteria: Bifunctional signals in virulence and host recognition. Curr. Opin. Microbiol. 3:73-78.

Kovach, M. E., Elzer, P. H., Hill, D. S., Robertson, G. T., Farris, M. A., Roop, R. M., 2nd, and Peterson, K. M. 1995. Four new derivatives of the broad-host-range cloning vector pBBR1MCS, carrying different antibiotic-resistance cassettes. Gene 166:175-176.

Laemmli, U. K. 1970. Cleavage of structural proteins during the assembly of the head of bacteriophage T4. Nature 227:680-685.

Lee, J. H., Choi, J. M., Lee, C., Yi, K. J., and Cho, Y. 2005. Structure of a peptide:N-glycanase-Rad23 complex: Insight into the deglycosylation for denatured glycoproteins. Proc. Natl. Acad. Sci. U.S.A. 102:91449149.

Li, G., Zhou, X., Zhao, G., Schindelin, H., and Lennarz, W. J. 2005. Multiple modes of interaction of the deglycosylation enzyme, mouse peptide N-glycanase, with the proteasome. Proc. Natl. Acad. Sci. U.S.A. 102:15809-15814.

Lindeberg, M., Stavrinides, J., Chang, J. H., Alfano, J. R., Collmer, A., Dangl, J. L., Greenberg, J. T., Mansfield, J. W., and Guttman, D. S. 2005. Proposed guidelines for a unified nomenclature and phylogenetic analysis of type III Hop effector proteins in the plant pathogen Pseudomonas syringae. Mol. Plant Microbe-Interact. 18:275-282.

Lopez-Solanilla, E., Bronstein, P. A., Schneider, A. R., and Collmer, A. 2004. HopPtoN is a Pseudomonas syringae Hrp (type III secretion system) cysteine protease effector that suppresses pathogen-induced necrosis associated with both compatible and incompatible plant interactions. Mol. Microbiol. 54:353-365.

Lorand, L., and Graham, R. M. 2003. Transglutaminases: Crosslinking enzymes with pleiotropic functions. Nat. Rev. Mol. Cell Biol. 4:140-156.

Mackey, D., Holt, B. F., 3rd, Wiig, A., and Dangl, J. L. 2002. RIN4 interacts with Pseudomonas syringae type III effector molecules and is required for RPM1-mediated resistance in Arabidopsis. Cell 108:743-754.

Mackey, D., Belkhadir, Y., Alonso, J. M., Ecker, J. R., and Dangl, J. L. 2003. Arabidopsis RIN4 is a target of the type III virulence effector AvrRpt2 and modulates RPS2-mediated resistance. Cell 112:379-389.

Makarova, K. S., Aravind, L., and Koonin, E. V. 1999. A superfamily of archaeal, bacterial, and eukaryotic proteins homologous to animal transglutaminases. Protein Sci. 8:1714-1719.

Mansfield, J., Jenner, C., Hockenhull, R., Bennett, M. A., and Stewart, R. 1994. Characterization of avrPphE, a gene for cultivar-specific avirulence from Pseudomonas syringae pv. phaseolicola which is physically linked to hrpY, a new hrp gene identified in the halo-blight bacterium. Mo.1 Plant-Microbe Interact. 7:26-739.

McNellis, T. W., Mudgett, M. B., Li, K., Aoyama, T., Horvath, D., Chua, N. H., and Staskawicz, B. J. 1998. Glucocorticoid-inducible expression of a bacterial avirulence gene in transgenic Arabidopsis induces hypersensitive cell death. Plant J. 14:247-257.

Mudgett, M. B. 2005. New insights to the function of phytopathogenic bacterial type III effectors in plants. Annu Rev Plant Biol 56:509-531.

Nagai, H., Cambronne, E. D., Kagan, J. C., Amor, J. C., Kahn, R. A., and Roy, C. R. 2005. A C-terminal translocation signal required for Dot/Icm-dependent delivery of the Legionella RalF protein to host cells. Proc. Natl. Acad. Sci. U.S.A. 102:826-831.

Nimchuk, Z., Marois, E., Kjemtrup, S., Leister, R. T., Katagiri, F., and Dangl, J. L. 2000. Eukaryotic fatty acylation drives plasma membrane targeting and enhances function of several Type III effector proteins from Pseudomonas syringae. Cell 101:353-363.

Nimchuk, Z., Rohmer, L., Chang, J. H., and Dangl, J. L. 2001. Knowing the dancer from the dance: R-gene products and their interactions with other proteins from host and pathogen. Curr. Opin. Plant Biol. 4:288294.

Nimchuk, Z., Eulgem, T., Holt, B. F., III, and Dangl, J. L. 2003. Recognition and response in the plant immune system. Annu. Rev. Genet. 37:579-609.

Nomura, K., Melotto, M., and He, S. Y. 2005. Suppression of host defense in compatible plant-Pseudomonas syringae interactions. Curr. Opin. Plant Biol. 8:361-368.

Orth, K., Xu, Z., Mudgett, M. B., Bao, Z. Q., Palmer, L. E., Bliska, J. B., Mangel, W. F., Staskawicz, B., and Dixon, J. E. 2000. Disruption of signaling by Yersinia effector YopJ, a ubiquitin-like protein protease. Science 290:1594-1597.

Pautsch, A., Vogelsgesang, M., Trankle, J., Herrmann, C., and Aktories, K. 2005. Crystal structure of the C3bot-RalA complex reveals a novel type of action of a bacterial exoenzyme. EMBO (Eur. Mol. Biol. Organ.) J. 24, 3670-3680.

Rea, P. A. 2006. Phytochelatin synthase, papain's cousin, in stereo. Proc Natl. Acad. Sci. U.S.A. 103:507-508.

Robert-Seilaniantz, A., Shan, L., Zhou, J. M., and Tang, X. 2006. The Pseudomonas syringae pv. tomato DC3000 Type III effector HopF2 has a putative myristoylation site required for its avirulence and virulence functions. Mol. Plant-Microbe Interact. 19:130-138.

Rohmer, L., Kjemtrup, S., Marchesini, P., and Dangl, J. L. 2003. Nucleotide sequence, functional characterization and evolution of $\mathrm{pFKN}$, a virulence plasmid in Pseudomonas syringae pathovar maculicola. Mol. Microbiol. 47:1545-1562.

Romanyuk, N. D., Rigden, D. J., Vatamaniuk, O. K., Lang, A., Cahoon, R. E., Jez, J. M., and Rea, P. A. 2006. Mutagenic definition of papain-like catalytic triad, sufficiency of N-terminal domain for single-site core catalytic enzyme acylation and C-terminal domain for augmentative metal activation of an eukaryotic phytochelatin synthase. Plant Physiol. 141:858-869.

Salanoubat, M., Genin, S., Artiguenave, F., Gouzy, J., Mangenot, S., Arlat, M., Billault, A., Brottier, P., Camus, J. C., Cattolico, L., et al. 2002. Genome sequence of the plant pathogen Ralstonia solanacearum. Nature 415:497-502.

Schechter, L. M., Roberts, K. A., Jamir, Y., Alfano, J. R., and Collmer, A. 2004. Pseudomonas syringae type III secretion system targeting signals and novel effectors studied with a Cya translocation reporter. J. Bacteriol. 186:543-555.

Shan, L., Thara, V. K., Martin, G. B., Zhou, J.-M., and Tang, X. 2000. The Pseudomonas AvrPto protein is differentially recognized by tomato and tobacco and is localized to the plant plasma membrane. Plant Cell 12:2323-2337.

Shao, F., Merritt, P. M., Bao, Z., Innes, R. W., and Dixon, J. E. 2002. A Yersinia effector and a Pseudomonas avirulence protein define a family of cysteine proteases functioning in bacterial pathogenesis. Cell 109:575-588.

Shao, F., Golstein, C., Ade, J., Stoutemyer, M., Dixon, J. E., and Innes, R. W. 2003. Cleavage of Arabidopsis PBS1 by a bacterial type III effector. Science 301:1230-1233

Sorg, J. A., Miller, N. C., and Schneewind, O. 2005. Substrate recognition of type III secretion machines--testing the RNA signal hypothesis. Cell Microbiol. 7:1217-1225.

Stevens, C., Bennett, M. A., Athanassopoulos, E., Tsiamis, G., Taylor, J. D., and Mansfield, J. W. 1998. Sequence variations in alleles of the avirulence gene avrPphE.R2 from Pseudomonas syringae pv. phaseolicola lead to loss of recognition of the AvrPphE protein within bean cells and a gain in cultivar-specific virulence. Mol. Microbiol. 29:165-177.

Suzuki, T., Park, H., and Lennarz, W. J. 2002. Cytoplasmic peptide:N-glycanase (PNGase) in eukaryotic cells: Occurrence, primary structure, and potential functions. FASEB (Fed. Am. Soc. Exp. Biol.) J. 16:635641.

Warren, R. F., Merritt, P. M., Holub, E., and Innes, R. W. (1999). Identification of three putative signal transduction genes involved in $\mathrm{R}$ genespecified disease resistance in Arabidopsis. Genetics 152:401-412.

\section{AUTHOR-RECOMMENDED INTERNET RESOURCE}

University of Dundee JPRED program: www.compbio.dundee.ac.uk/ www-jpred 